\title{
Uso de Rejeito de Lavagem de Bauxita para a Fabricação de Ligantes Geopoliméricos
}

\author{
Use of Bauxite Washout Reagent for the \\ Manufacture of Geopolymer Binders
}

Lêda de Azevedo Racanelli ${ }^{1}$, Raíza Cavalcante de Oliveira ${ }^{2}$, Woshington da Silva Brito ${ }^{2}$, José Antônio da Silva Souza ${ }^{2}$

\author{
${ }^{1}$ UFPA/ITEC/PRODERNA - Programa de Pós-Graduação em Engenharia de Recursos Naturais da Amazônia, CEP: \\ 66075-110, Belém, PA, Brasil. \\ e-mail: lbsracanelli@hotmail.com \\ ${ }^{2}$ UFPA/ITEC/Departamento de Engenharia de Processos, Belém, PA, Brasil. \\ e-mail: raiza_cavalcante@hotmail.com,wsbrito3@gmail.com,jass@ufpa.br
}

\begin{abstract}
RESUMO
A realidade negativa dos impactos ambientais tem feito com que a sociedade busque por alternativas menos agressivas ao meio ambiente. As cadeias produtivas das grandes empresas, infelizmente, são geradoras de resíduos ou rejeitos que contribuem para essa realidade que com mais frequência tem se tornado foco de discussão. Essa questão ambiental passa a ser de extrema importância para a sociedade do estado do Pará, uma vez que possui grande potencial de mineração voltado para produção de alumínio por exemplo, e que naturalmente pelo seu processo, produz resíduos e rejeitos que são depositados em forma de grandes lagoas. Vislumbrando uma contribuição positiva para sociedade e meio ambiente, optou-se por utilizar o rejeito da lavagem da bauxita como fonte de aluminossilicatos para produção de geopolímeros que são materiais cimentícios com estrutura tridimensional formados por ativação alcalina. Portanto, esta pesquisa tem como objetivo verificar a possibilidade de transformar o rejeito da bauxita em um ligante geopolimérico de resistência mecânica equiparada ao cimento do tipo Portland. Com razão de Davidovits $\left(\mathrm{SiO}_{2} / \mathrm{Al}_{2} \mathrm{O}_{3}\right)$ de 0,81 e 0,82 para as respectivas temperaturas de calcinação à $600^{\circ} \mathrm{C}$ e $700^{\circ} \mathrm{C}$, testes à compressão foram avaliados por 3 e 10 dias de cura. A reação de geopolimerização foi conduzida a temperatura ambiente de $28^{\circ} \mathrm{C}$ utilizando como ativadores hidróxido de sódio 15 molar e silicato de sódio alcalino 10 molar. Utilizou-se técnicas de DRX, FRX, MEV, Análise granulométrica, TG e ATD para caracterização das matérias-primas e dos geopolímeros. O melhor geopolímero ficou com matéria-prima calcinada à $700^{\circ} \mathrm{C}$, de maior razão Davidovits 0,82 , apresentando trabalhabilidade no seu estado fresco e maior resistência mecânica à compressão acima de 10,0MPa para 3 dias, quando comparado às normas para o cimento do tipo CP II E 32.
\end{abstract}

Palavras-chave: geopolímero; rejeito da bauxita; ativador alcalino; resistência a compressão.

\section{ABSTRACT}

The negative reality of environmental impacts has made society look for less aggressive alternatives to the environment. The productive chains of large companies, unfortunately, generate waste or tailings that contribute to this reality that has become the focus of discussion. This environmental issue becomes extremely important for the society of the state of Pará, since it has great mining potential focused on aluminum production, for example, and naturally by its process, produces waste and tailings that are deposited in form of large ponds. Aiming to make a positive contribution to society and the environment, we chose to use bauxite washing tailings as a source of aluminosilicates for the production of geopolymers that are three-dimensional cementitious materials formed by alkaline activation. Therefore, this research aims to verify the possibility of transforming bauxite tailings into a geopolymeric binder of mechanical strength equivalent to Portland cement. With Davidovits ratio $\left(\mathrm{SiO}_{2} / \mathrm{Al}_{2} \mathrm{O}_{3}\right)$ of 0.81 and 0.82 for the respective calcination temperatures at $600^{\circ} \mathrm{C}$ and $700^{\circ} \mathrm{C}$, pressure tests were evaluated for 3 and 10 days of cure. The geopolymerization reaction was conducted at room temperature of $28^{\circ} \mathrm{C}$ using as activators 15 molar sodium hydroxide and 10 molar alkaline sodium silicate. XRD, XRF, SEM, particle size analysis, TG and DTA techniques were used to char- 
acterize the raw materials and geopolymers. The best geopolymer was calcined raw material at $700^{\circ} \mathrm{C}, \mathrm{Da}-$ vidovits highest ratio 0.82 , presenting fresh workability and greater mechanical compressive strength above 10.0MPa for 3 days when compared to the standards for CP II E 32 type cement.

Keywords: geopolymer; bauxite tailings; alkaline activator; resistance to compression.

\section{INTRODUÇÃO}

Devido seu potencial mineral, o estado do Pará destaca-se representando uma liderança de aproximadamente $90 \%$ na produção de caulim para cobertura de papel e bauxita metalúrgica [1]. Se tratando da bauxita metalúrgica, há todo um processo de produção que não será aprofundado nesta pesquisa, mas que será mencionada apenas a primeira etapa que já se inicia com a geração de rejeitos industriais.

A obtenção do alumínio comercial é a última etapa de todo o processo, e ao longo dela são gerados rejeitos os quais são depositados no meio ambiente, normalmente dispostos em lagoas projetadas especialmente para este fim. Vale ressaltar que não se trata da lama vermelha, que é um resíduo insolúvel gerado durante a etapa de clarificação do processo Bayer [2], mas sim da primeira etapa de beneficiamento da bauxita que consiste em separar a caulinita através do processo de desagregação/lavagem [1].

Esse rejeito também é depositado em lagoas projetadas, rico em Si e Al, possuindo também o argilomineral caulinita em sua constituição, apresenta-se como uma fonte alternativa para estudos de produção/aplicação de materiais geopoliméricos.

Pode-se dizer, dentre alguns vários outros conceitos, que geopolímero é uma classe de ligante de polímero inorgânico tipicamente sintetizado através de ativação alcalina de precursores de aluminossilicatos, possuem boa resistência à alta temperatura e à degradação de ácido, bem como boas propriedades mecânicas [3]. Com tais propriedades torna-se uma alternativa em busca de substituir o cimento do tipo Portland, além do que seu uso permite à reciclagem de grandes quantidades de resíduos ou rejeitos industriais [4].

Como a geopolimerização ocorre dessa relação $\mathrm{SiO}_{2} / \mathrm{Al}_{2} \mathrm{O}_{3}$ pode-se dizer que obter um material geopolimérico é particularmente complexo devido à cinética da reação, mas a maioria dos autores concorda que o mecanismo envolve a dissolução, seguida de policondensação de gel [5].

Apesar dessa nova classe de ligante ter surgido na década de 1970, vários estudos já comprovaram a eficiência e mais ainda, mostraram que as propriedades mecânicas dos geopolímeros aumentam com a utilização de metacaulim (fase amorfa do mineral caulim) [6,7]. Então, por ser um material ainda relativamente novo no âmbito de estudo e desenvolvimento, ele não possui normas técnicas próprias para efetuação de seus ensaios. Por conta disso, utilizou-se normas do material cimentício de comportamento mais próximo, para verificar a possibilidade de transformar o rejeito da bauxita em um ligante geopolimérico de resistência mecânica equiparada a cimento do tipo Portland.

\section{MATERIAIS E MÉTODOS}

O rejeito da lavagem da bauxita $(\mathrm{RB})$ foi coletado via úmido na mina de Miltônia, localizada no município de Paragominas, estado do Pará. Esse resíduo foi utilizado como fonte de Si e Al para síntese de geopolímero. Para aumentar a reatividade da reação de geopolimerização, este material passou por processo térmico em temperaturas de $600 \mathrm{e} 700^{\circ} \mathrm{C}$ por duas horas.

Para as análises das fases minerais presentes no rejeito da bauxita "in natura", foi utilizado difratômetro de raios X (DRX) de marca Empyrean da PANalytical, operando a $40 \mathrm{kV}$ e $35 \mathrm{~mA}$ e com um comprimento de onda $\lambda \mathrm{CoK} \alpha=1,78 \AA$. A amostra foi examinada em um intervalo de $2 \theta$ entre $3-95^{\circ}$, com um passo angular de $0,02^{\circ}$ e tempo por passo $27 \mathrm{~s}$. Para análise química usou-se a fluorescência de raios $\mathrm{X}$ por energia dispersiva (EDX) em um equipamento Shimadzu, modelo EDX-700. As análises de ATD/TG foram realizadas em um equipamento modelo PL Thermal Science com analisador térmico simultâneo, da Stanton Redcroft, com forno cilíndrico vertical, conversor digital acoplado a um microcomputador, utilizando uma faixa de temperatura entre 30 e $1000^{\circ} \mathrm{C}$, com taxa de aquecimento de $10^{\circ} \mathrm{C}$.min- 1 em atmosfera estática do ar. Para análise granulométrica utilizou-se $1 \mathrm{~g}$ da amostra colocada em suspensão em $7 \mathrm{ml}$ de água destilada. Em seguida, o líquido com a amostra é pipetado em uma unidade de dispersão que também utiliza água como líquido de suspensão. A leitura foi realizada no analisador de partículas a laser marca Fritsch $\mathrm{GmbH}$, modelo Analysette 22 MicroTec Plus., com intervalo de medida de 0,08 a $2.000 \mu \mathrm{m}$. Para análise do MEV e EDS utilizou-se dos fragmentos de todos os geopolímeros submetidos ao ensaio de compressão, em equipamento de marca HITACHI, modelo TM 3000. Para o ensaio de resistência a compressão utilizou-se a máquina universal Emic SSH300, célula Trd 30, com velocidade de 0,25MPa/s de acordo com a norma ABNT NBR 13279.

Para síntese do geopolímero utilizou-se ativadores alcalinos compostos de uma solução de hidróxido 
de sódio $(\mathrm{NaOH})$ micro perola (Neon, $97 \%$ pureza) e solução de silicato de sódio alcalino $\left(\mathrm{Na}_{2} \mathrm{SiO}_{3}\right)(\mathrm{Man}-$ chester Química do Brasil S.A., $\mathrm{SiO}_{2} / \mathrm{Na}_{2} \mathrm{O}=3,20$ ). Os ativadores alcalinos foram preparados com $24 \mathrm{~h}$ de antecedência para se evitar aquecimento durante a dissolução do $\mathrm{NaOH}$, o que poderia afetar as reações de geopolimerização.

Ainda não existe normas regulamentadoras para materiais geopoliméricos, mas, devido a característica cimentícia apresentada por esta nova classe de material, utiliza-se como parâmetro de comparação as normas aplicáveis à indústria do cimento.

A escolha da variação do hidróxido de sódio baseia-se no fato de que não se encontra na literatura ligante geopolimérico desenvolvidos com a primeira lavagem da bauxita (RB) nessas variações, por isso a escolha para os devidos testes. A Tabela 1 apresenta a variação da concentração dos ativadores utilizados.

Tabela 1: Concentração molar e proporções da solução ativadora.

\begin{tabular}{l|c|c}
\hline \multicolumn{1}{c|}{ ATIVADOR: } & $\mathrm{NaOH}$ & $\mathrm{Na}_{2} \mathrm{SiO}_{3}$ \\
\hline \multirow{3}{*}{ CONCENTRAÇÃO MOLAR } & 05 & 10 \\
\cline { 2 - 3 } & 15 & 10 \\
\cline { 2 - 3 } & 20 & 10 \\
\cline { 2 - 3 } & 25 & 10 \\
\cline { 2 - 3 } & 30 & 10 \\
\hline PROPORÇÃO $\left(\mathrm{NaOH}: \mathrm{Na}_{2} \mathrm{SiO}_{3}\right)$ & \multicolumn{2}{|c|}{$1: 3$ EM MASSA DE SOLUÇÃO } \\
\hline
\end{tabular}

A elaboração da pasta geopolimérica inicia-se pela massa do rejeito adicionada em recipiente (peça componente do misturador) concomitantemente os ativadores também são adicionados, a saber, $\mathrm{NaOH}$ e $\mathrm{Na}_{2} \mathrm{SiO}_{3}$. Após 2 minutos no misturador mecânico, as pastas frescas foram transferidas para moldes plásticos cilíndricos com dimensões de $100 \mathrm{~mm}$ x50 mm (altura x diâmetro). Posteriormente, foram submetidos à cura em temperatura ambiente de $28^{\circ} \mathrm{C}$ com testes de resistência à compressão para 3 e 10 dias de cada concentração. Cinco corpos de prova de cada condição foram utilizados para as medidas das resistências à compressão.

\section{RESULTADOS E DISCUSSÃO}

A Figura 1 apresenta o resultado da análise granulométrica realizada no rejeito da bauxita in natura.

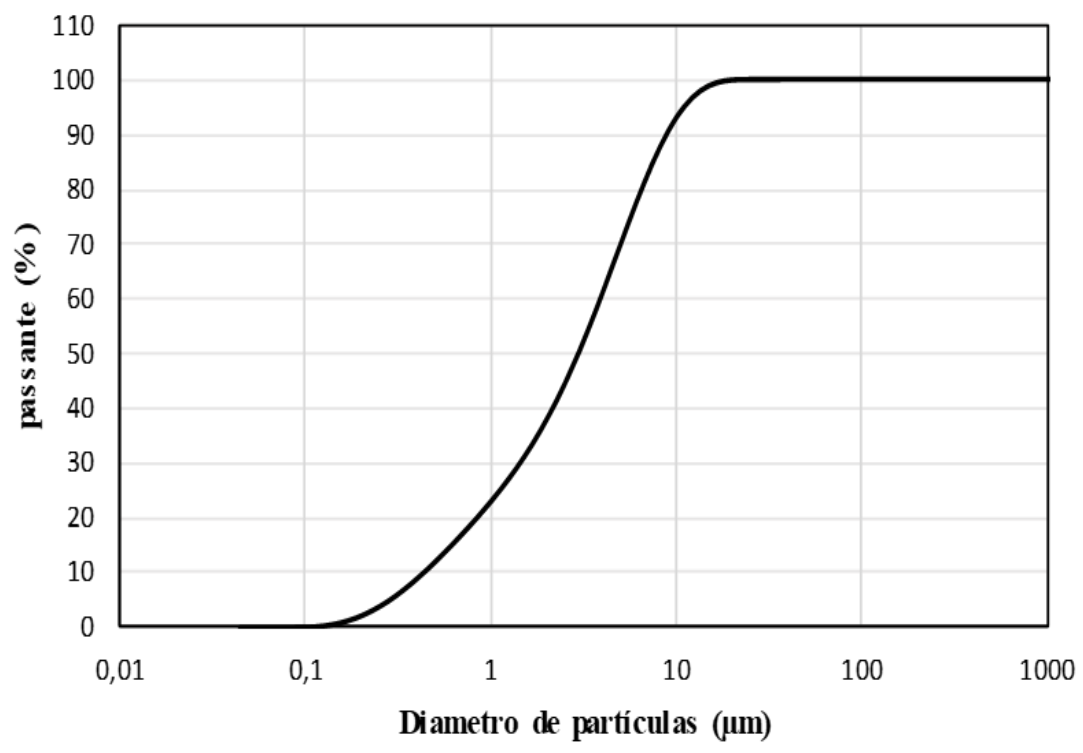

Figura 1: Diâmetro das partículas do R.B

A distribuição de tamanho de partícula resultou em um diâmetro médio $\left(\mathrm{d}_{50}\right)$ de $3,1 \mu \mathrm{m}$, variando entre 0,5 a $9,3 \mu \mathrm{m}$, por conta disso, pode-se dizer que esse rejeito é classificado como uma suspensão formada por partículas ultrafinas [8], pois a distribuição granulométrica indicou que cerca de 78,5\% das partículas possuem tamanhos menores que $0,87 \mu \mathrm{m}, 49,18 \%$ encontram-se abaixo de $2,86 \mu \mathrm{m}$ e $4,97 \%$ estão abaixo de 
$10,42 \mu \mathrm{m}$.

O comportamento térmico do RB é mostrado na Figura 2. Na curva TG, observa-se que o material, quando aquecido até $1000^{\circ} \mathrm{C}$ apresenta uma perda de massa total de $7,1 \%$.

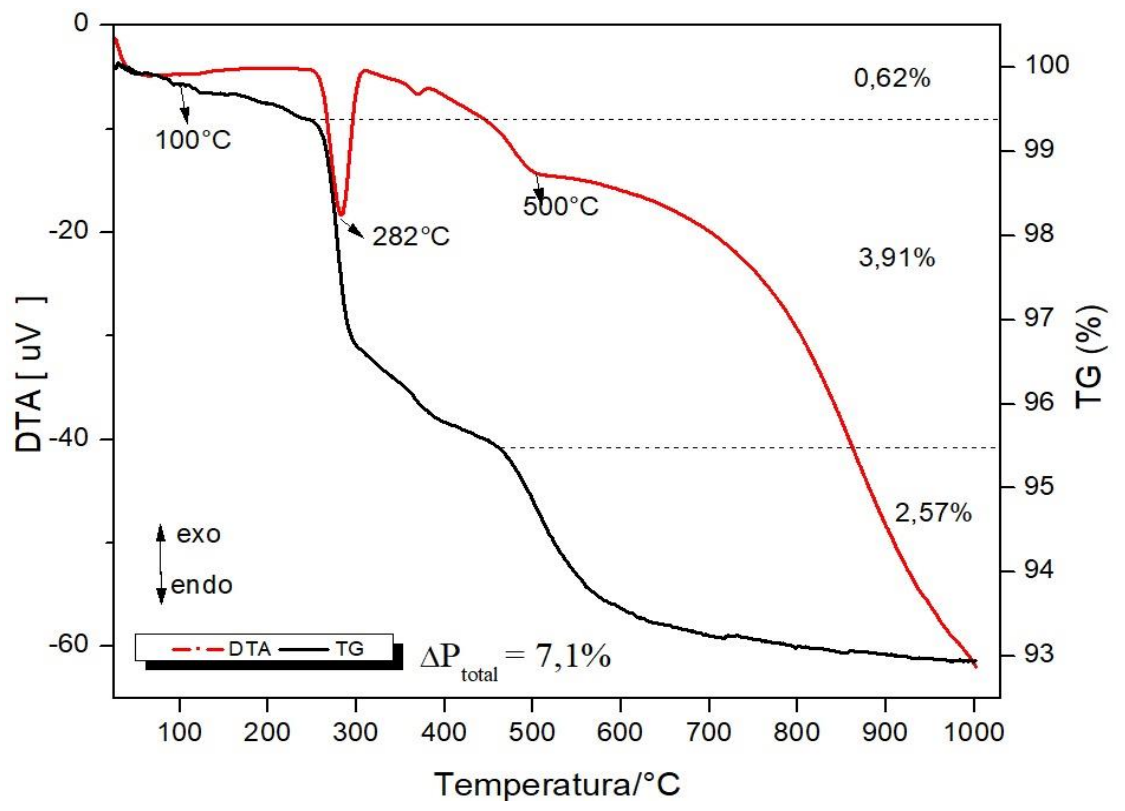

Figura 2: DTA/TG do R.B

Como pode ser observado na Figura 2, o material apresenta um evento em torno de $100^{\circ} \mathrm{C}$, refere-se à perda de água de hidratação presente na bauxita [9]. Pode-se observar também que a curva DTA apresenta uma intensa endoterma na faixa de temperatura entre 220 a $300^{\circ} \mathrm{C}$, o que corresponde à transformação da gibsita em boemita. Entre 300 e $520^{\circ} \mathrm{C}$, ocorre a segunda etapa de perda de massa, a qual se refere à desidroxilação da fase boemita levando à formação de óxidos de alumínio metaestáveis [10,11,12].

A Figura 3 apresenta o resultado da análise de DRX do rejeito da bauxita in natura. Segundo [13] o minério de bauxita consiste em uma mistura de minerais de hidróxido (gibsita, boemita e diásporo) e impurezas (principalmente caulinita, hematita, goetita, quartzo e anatásio). O resultado da análise demonstra que o rejeito é do tipo gibisitico com fases predominantes como: Gibsita, óxidos de ferro e caulinita.

Todas as identificações foram indexadas de acordo com as cartas cristalográficas: Gibsita (PDF 01070-2038); Caulinita (PDF 01-083-0971); Anatásio (PDF 01-071-1166) e Hematita (PDF 01-085-0599).

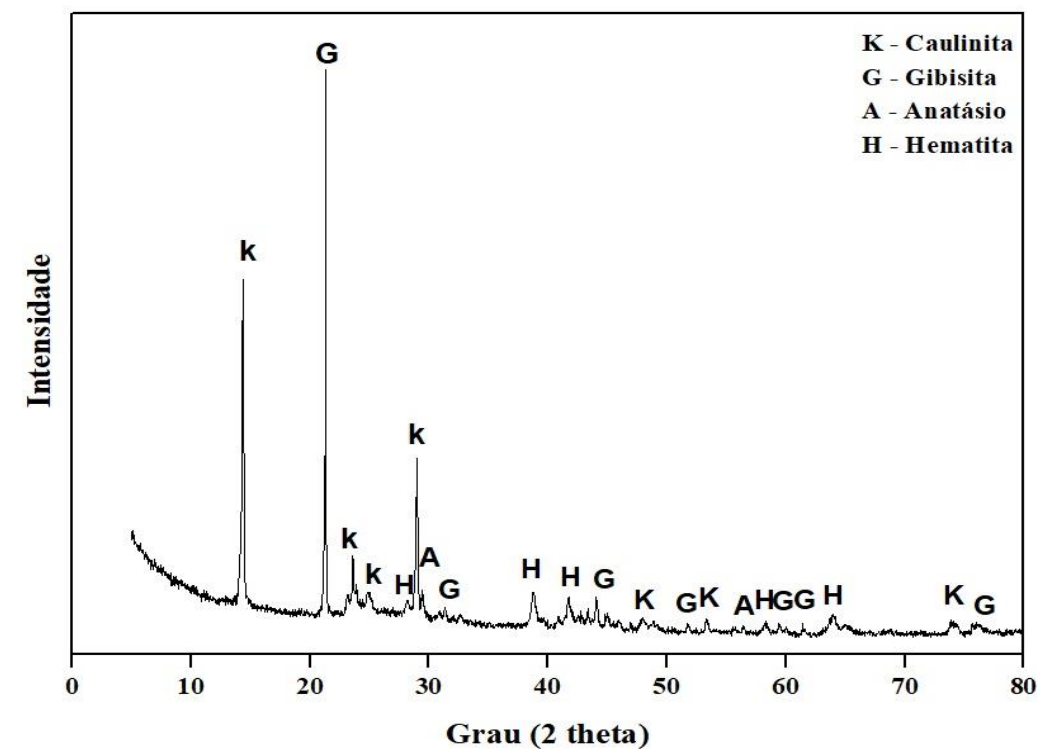

Figura 3: DRX do rejeito da bauxita (RB) 
Aluminossilicatos naturais, quando submetidos a um tratamento térmico, sofrem um processo de desidroxilação e ocorre alteração da coordenação do alumínio, dando lugar à obtenção do metacaulim, que pode também ser ativado alcalinamente $[14,15]$. Em vista disso, buscou-se verificar o comportamento desse material partindo das temperaturas de $500^{\circ} \mathrm{C}, 600^{\circ} \mathrm{C}$ até $700^{\circ} \mathrm{C}$. A Figura 4 apresenta a análise de DRX em todas essas temperaturas.

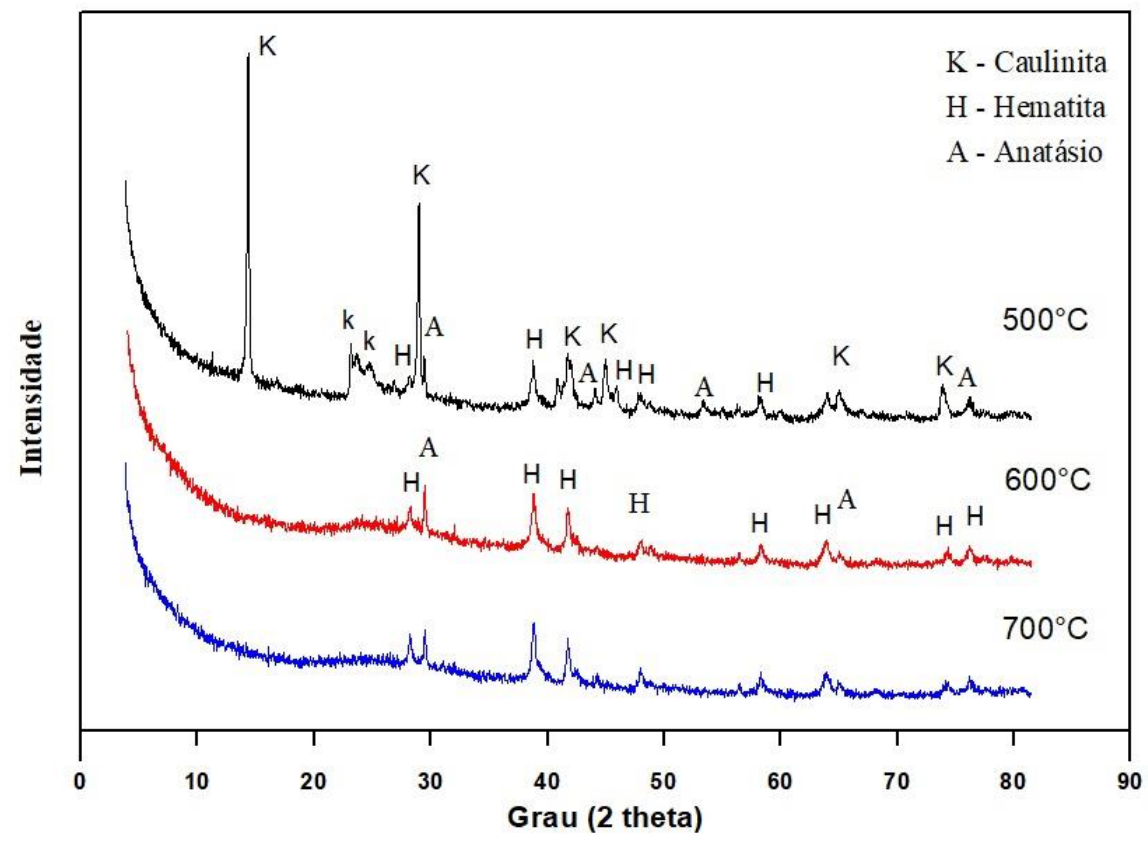

Figura 4: DRX do rejeito da bauxita $(\mathrm{RB})$ em $500^{\circ} \mathrm{C}, 600^{\circ} \mathrm{C}$ e $700^{\circ} \mathrm{C}$

Analisando a $500^{\circ} \mathrm{C}$ percebe-se a presença de várias fases cristalinas, porém como fase amplamente majoritária a caulinita. A partir de $580^{\circ} \mathrm{C}$ a caulinita inicia a formação da metacaulinita amorfa [12], portanto, impossibilitando, ainda nessa temperatura de $500^{\circ} \mathrm{C}$, sua utilização para produção de material geopolimérico, uma vez que estudos mostram que as forças mecânicas dos geopolímeros aumentam com a elevação da temperatura de calcinação [16,17].

Quando as amostras são submetidas a $600^{\circ} \mathrm{C}$ e $700^{\circ} \mathrm{C}$ o comportamento das fases cristalinas é muito similar, ou seja, a partir de $600^{\circ} \mathrm{C}$ percebe-se que a caulinita desaparece dando lugar a uma fase amorfa que só irá se recristalinizar formando a mulita a partir de $980^{\circ} \mathrm{C}$ [12]. Então, nas duas últimas temperaturas já se obtêm um material amorfo com fases como hematita e anatásio de pouca intensidade.

A Tabela 2 apresenta os resultados das análises de EDX do rejeito utilizado para síntese do geopolímero. Observa-se que o teor dos óxidos como sílica e alumina para a temperaturas de $600^{\circ} \mathrm{C}$ e $700^{\circ} \mathrm{C}$, indicam ser uma possível fonte de Al e Si na produção de materiais geopoliméricos [18].

Tabela 2: Composição química em $\%$ do rejeito da bauxita em $600^{\circ} \mathrm{C}$ e $700^{\circ} \mathrm{C}$, respectivamente.

\begin{tabular}{ccccccccc}
\hline MATERIAL & $\mathrm{SiO}_{2}$ & $\mathrm{Al}_{2} \mathrm{O}_{3}$ & $\mathrm{Na}_{2} \mathrm{O}$ & $\mathrm{K}_{2} \mathrm{O}$ & $\mathrm{MgO}$ & $\mathrm{CaO}$ & $\mathrm{Fe}_{2} \mathrm{O}_{3}$ & $\mathrm{TiO}_{2}$ \\
\hline $\mathrm{RB}-600^{\circ} \mathrm{C}$ & 23,09 & 48,34 & - & - & - & - & 25,44 & 3,13 \\
\hline $\mathrm{RB}-700^{\circ} \mathrm{C}$ & 24,09 & 49,65 & - & - & - & 0,32 & 22,66 & 2,88 \\
\hline
\end{tabular}

De acordo com a análise, percebe-se que em seu estado amorfo o rejeito apresenta sílica e alumina em altos teores. Quanto aos óxidos, $\mathrm{Na}_{2} \mathrm{O}, \mathrm{K}_{2} \mathrm{O}$ e $\mathrm{MgO}$, nota-se que não fazem parte da composição do rejeito da bauxita e nem estão associados a nenhum tipo de mineral nos difratogramas. A concentração de $\mathrm{Fe}_{2} \mathrm{O}_{3}$ observada na fluorescência está de acordo com os estudos de [19] e apresenta-se também nos difratogramas como nas fases de hematita.

A Figura 5 apresenta o pré-teste feito no RB à $600^{\circ} \mathrm{C}$ em malha passante de \#65 $(210 \mu \mathrm{m})$. 


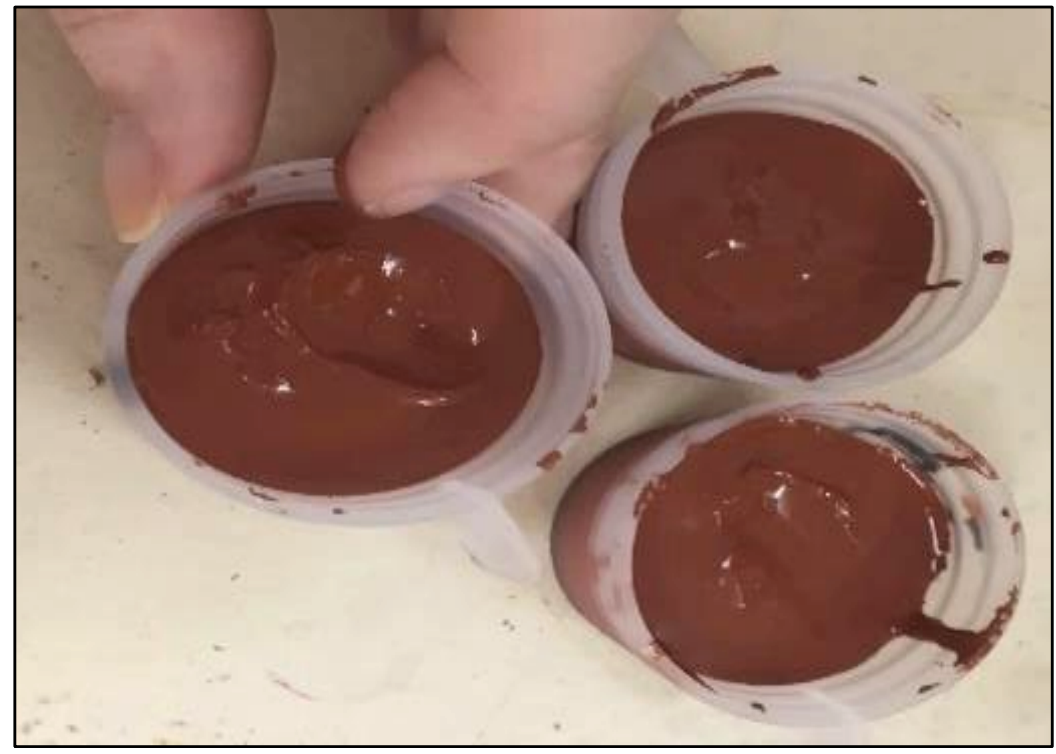

Figura 5: RB à $600^{\circ} \mathrm{C}$ após 10 dias

Como pode-se notar, passados os 10 dias de cura o material ainda se apresentava úmido, sem condições de efetuar os testes propostos por esta pesquisa. Logo, fica a sugestão de uma pesquisa aprofundada sobre a geopolimerização do RB em temperatura de $600^{\circ} \mathrm{C}$.

A Figura 6 apresenta os resultados de resistência mecânica à compressão em MPa, e indica que o geopolímero ativado à uma concentração de 15 molar é o de maior resistência.

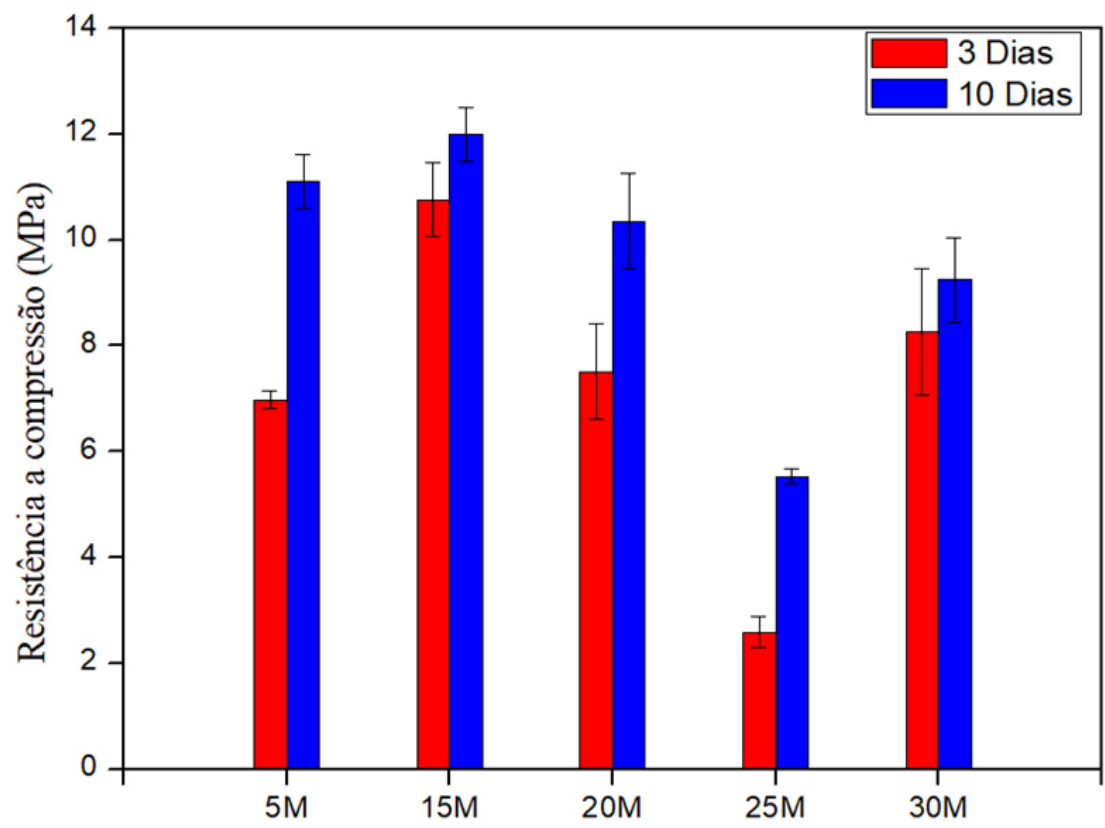

Figura 6: Resistência mecânica à compressão na temperatura de $700^{\circ} \mathrm{C}$

O geopolímero de 15 molar $(15 \mathrm{M})$ com razão de $0,82\left(\mathrm{SiO}_{2} / \mathrm{Al}_{2} \mathrm{O}_{3}\right)$ foi o que apresentou maior resistência à compressão atingindo valores de 10,76 e 12MPa para três e dez dias de cura, respectivamente, em temperatura ambiente. Pode-se observar ainda no gráfico que à medida que se aumenta a concentração dos ativadores, a resistência diminui e isso provavelmente pode estar relacionado com o excesso de hidróxido de sódio, que além de causar eflorescência, diminui a resistência mecânica e aumenta a porosidade já que, com a imediata reação de geopolimerização, ocasiona uma rápida cura [20].

A resistência do geopolímero, alcançado para três dias de cura (10 MPa), é igual ao valor estabelecido pela NBR 7215 ( CP II E 32), ou seja, obteve-se um material produzido com baixo consumo de energia, baixa emissão de $\mathrm{CO}_{2}$ e com características semelhantes às do cimento tipo Portland. 
A Figura 7 apresenta o MEV e EDS da estrutura do geopolímero de 15M após sofrer o ensaio de resistência à compressão.

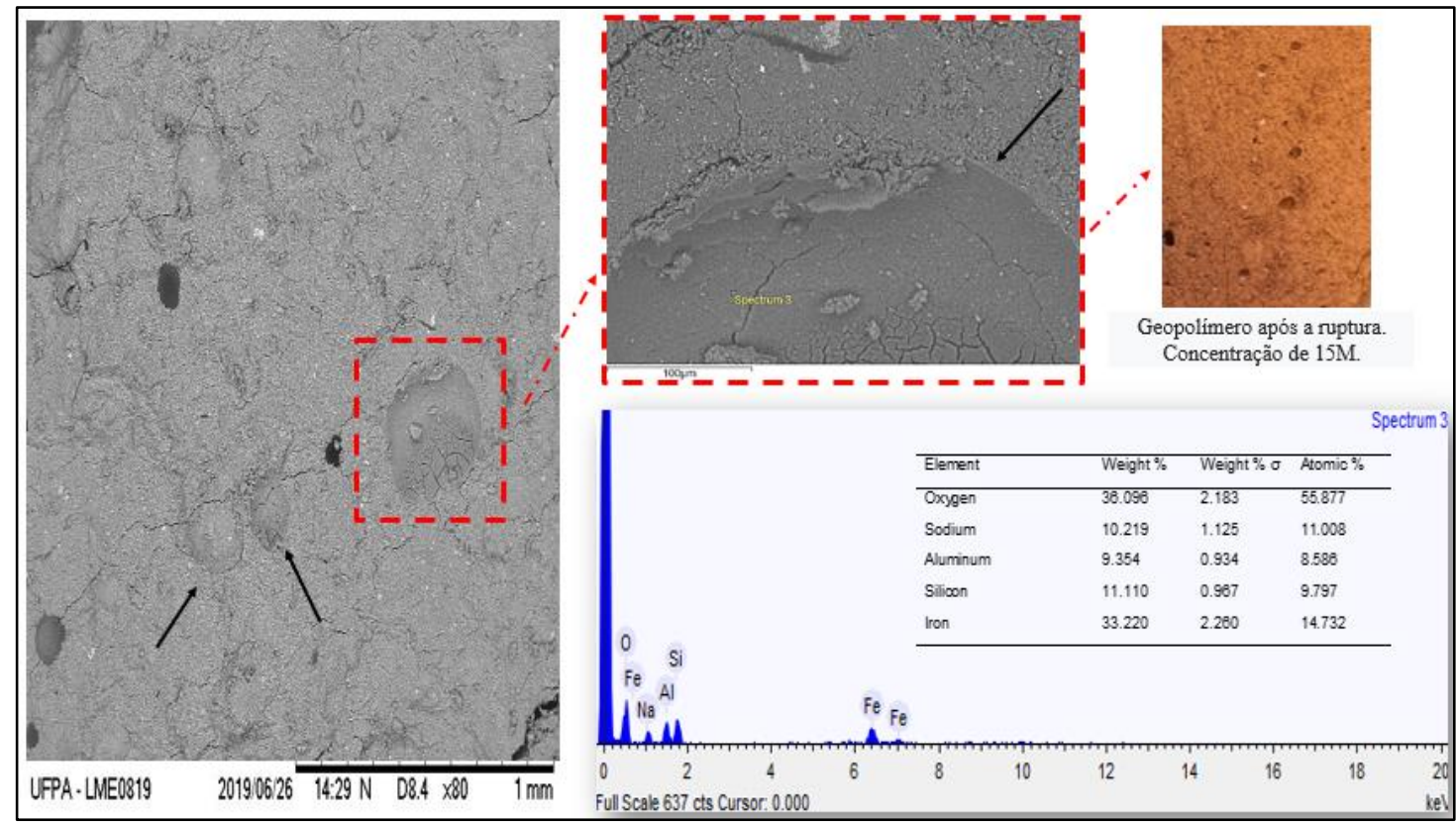

Figura 7: Geopolímero a 15M

Pela Figura 7 observa-se uma morfologia compactada, com poucas trincas e poros, o que the confere uma alta resistência à compressão em relação aos outros geopolímeros testados. Segundo [21] o aspecto morfológico mais denso e uniforme confere maior desempenho mecânico aos geopolímeros.

As setas pretas indicam pequenos círculos encontrados, estes no espectro do EDS são identificados como a presença do $\mathrm{Al}$ e Si mostrando o caráter de aluminossilicato da reação de geopolimerização, bem como a presença do Na que é devida ao ativador à base de hidróxido de sódio.

\section{CONCLUSÕES}

A pesquisa realizada pelos autores, permitiu concluir que não houve processo de geopolimerização com o rejeito da primeira lavagem da bauxita $(\mathrm{RB})$ à $600^{\circ} \mathrm{C}$. O RB possui diâmetro de partículas ultrafinas que contribuem no processo de aceleração da reação. A TG e DTA do RB in natura apresentou comportamento típico do mineral gibisitico comprovado através da análise por Difração de raios X. A caracterização por DRX na amostra do RB in natura revelou que são constituídas, sobretudo por Gibsita, Caulinita, Anatásio e Hematita. Em temperatura de $500^{\circ} \mathrm{C}$ apresenta fases como Caulinita, Anatásio e Hematita. A $600^{\circ} \mathrm{C}$ e $700^{\circ} \mathrm{C}$ apresentam estrutura parcialmente amorfa. A análise da resistência mostrou que apenas a concentração de $15 \mathrm{M}$ apresentou maior grau de reação de geopolimerização que foi indicado pela maior resistência à compressão. Notando-se que para esta concentração, em três dias de cura, obteve-se resistência mecânica iguais às do cimento tipo Portland (CP II E 32). E, a microscopia eletrônica de varredura apresentou um material compactado e com poucas regiões com trincas. Logo, o rejeito da primeira lavagem da bauxita mostrou ter grande potencial para produção de materiais geopoliméricos a partir de $700^{\circ} \mathrm{C}$. Importante ressaltar que os geopolímeros foram produzidos com baixa emissão de $\mathrm{CO}_{2}$, em relação aos processos de produção para os tipos convencionais, contribuindo assim de forma positiva ao meio ambiente.

\section{AGRADECIMENTOS}

Os autores agradecem a CAPES, PRODERNA, UFPA e a empresa pelo incentivo a pesquisa.

\section{BIBLIOGRAFIA}

[1] MELO, C. C. A., PAZ, S.P. A., ANGÉLICA, R. S. "Fases sodalita formadas a partir de caulinitas amazônicas sob condições de digestão Bayer”, Revista Matéria, ISSN 1517-7076, 2017. 
[2] SILVA FILHO, E. B., ALVES, M. C. M., MOTTA, M. "Lama vermelha da indústria de beneficiamento de alumina: produção, características, disposição e aplicações alternativas”, Revista Matéria, v. 12, n. 2, pp. $322-338,2007$.

[3] DAVIDOVITS, J., "Geopolymer and geopolymeric materials", J.THERM., pp. 429-441, https://doi.org/10.1017/CBO9781107415324.004, 1989.

[4] DUXSON, P., PROVIS, J.L., LUKEY, G.C., et al., "Understanding the relationship between geopolymer composition, microstructure and mechanical properties", Colloids and Surfaces A: Physicochemical and Engineering Aspects, v. 269, pp 47-58, 2005.

[5] DUXSON, P., FERNÁNDEZ-JIMÉNEZ, A., PROVIS, J. L., et al., "Geopolymer technology: the current state of the art", J. Mater. Sci., pp. 2917-2933, 2007.

[6] WANG, M.R., JIA D.C, HE, P.G., "Influence of calcination temperature of kaolin on the structure and properties of final geopolymer", Materials Letters, v.64, 2551-2554, 2010.

[7] CIOFFI, R., MAFFUCCI, L., SANTORO, L. Optimization of geopolymer synthesis by calcination anpolycondensation of a kaolinitic residue, Resour Conserv Recy, v.40, pp. 27-38. 2003.

[8] OLIVEIRA, C., RUBIO, "Mecanismos, técnicas e aplicações da agregação no tratamento mineral e ambiental”, J. Série Tecnologia Ambiental, v. 60. pp. 69, 2011.

[9] PRADO, C. M. R., ALVES, M. I. R., LELES, M. I. G., et al., "Estudo da ativação ácida e tratamento térmico de bauxita extraída de jazidas em Minas Gerais", Cerâmica, v. 58, pp. 111-117, 2012.

[10] BOUMAZA, A., DJELLOUL, A., GUERRAB, F., "Specific signatures of $\alpha$-alumina powders prepared by calcination of boehmiteor gibbsite", Powder Technology, v. 201, pp.177-180, 2010.

[11] ZHU, B., FANG, B., LI, X., Ceramics International, v.36, pp.2493-2498, 2010.

[12] SANTOS, P. S., Ciência e Tecnologia das Argilas, v.1, Editora Edgard Blücher, 1989.

[13] ANGÉLICA, R.S., KAHNB, H., PAZ, S.P.A. “A proposal for bauxite quality control using the combined Rietveld - Le Bail - Internal Standard PXRD method - Part 2: Application to a gibbsitic bauxite from the Paragominas region", Minerals Engineering, v.122, pp. 148-155, 2018.

[14] GRANIZO, M.L. “Activación Alcalina de Metacaolin: Desarrollo de Nuevos Materiales Cementantes", Tese de D.Sc., Universidade Autónoma de Madrid, 1998.

[15] MALKI, A., MEKHALIF, Z., DETRICHE, S., et al., "Calcination products of gibbsite studied by X-ray diffraction, XPS and solid-state NMR”, Journal of Solid State Chemistry, v. 215, pp. 8-15, 2014.

[16] JIAYUAN, Y.E., WENSHENG, Z., DI, S. "Properties of an aged geopolymer synthesized fromcalcined ore-dressing tailing of bauxite and slag", Cement and Concrete Research, v.100, pp.23-31, 2017.

[17] Y.J, N., DJOBO, A., ELIMBIA, A., et al., "Partial replacement of volcanic ash by bauxite and calcined oyster shell in the synthesis of volcanic ash-based geopolymers", Construction and Building Materials, v. 113, pp. 673-681, 2016.

[18] DAVIDOVITS, J. "Geopolymers: Inorganic Polymeric New Materials", Journal of Thermal Analysis, v.37, pp. 1633-1656, 1991.

[19] SAMPAIO, J. A., ANDRADE, M. C., DUTRA, A. J. B. Rochas e Minerais Industriais, CETEM, v.2, pp. 312-337, 2008

[20] NARAYANAN, A., SHANMUGASUNDARAM, P. “An Experimental Investigation on Flyash-based Geopolymer Mortar under different curing regime for Thermal Analysis", Energy and Buildings, v. 138, pp. 539-545, 2017.

[21] SALEHI, S., KHATTAK, M. J., BWALA, A. H., et al., "Characterization, morphology and shear bond strength analysis of geopolymers: Implications for oil and gas well cementing applications", Journal of Natural Gas Science and Engineering, v. 38, pp .323-332, 2017.

\section{ORCID}

Lêda de Azevedo Racanelli Raíza Cavalcante de Oliveira Woshington da Silva Brito José Antônio da Silva Souza https://orcid.org/0000-0002-0422-6803

https://orcid.org/0000-0002-5105-3001

https://orcid.org/0000-0002-2870-2042

https://orcid.org/0000-0002-1784-9217 


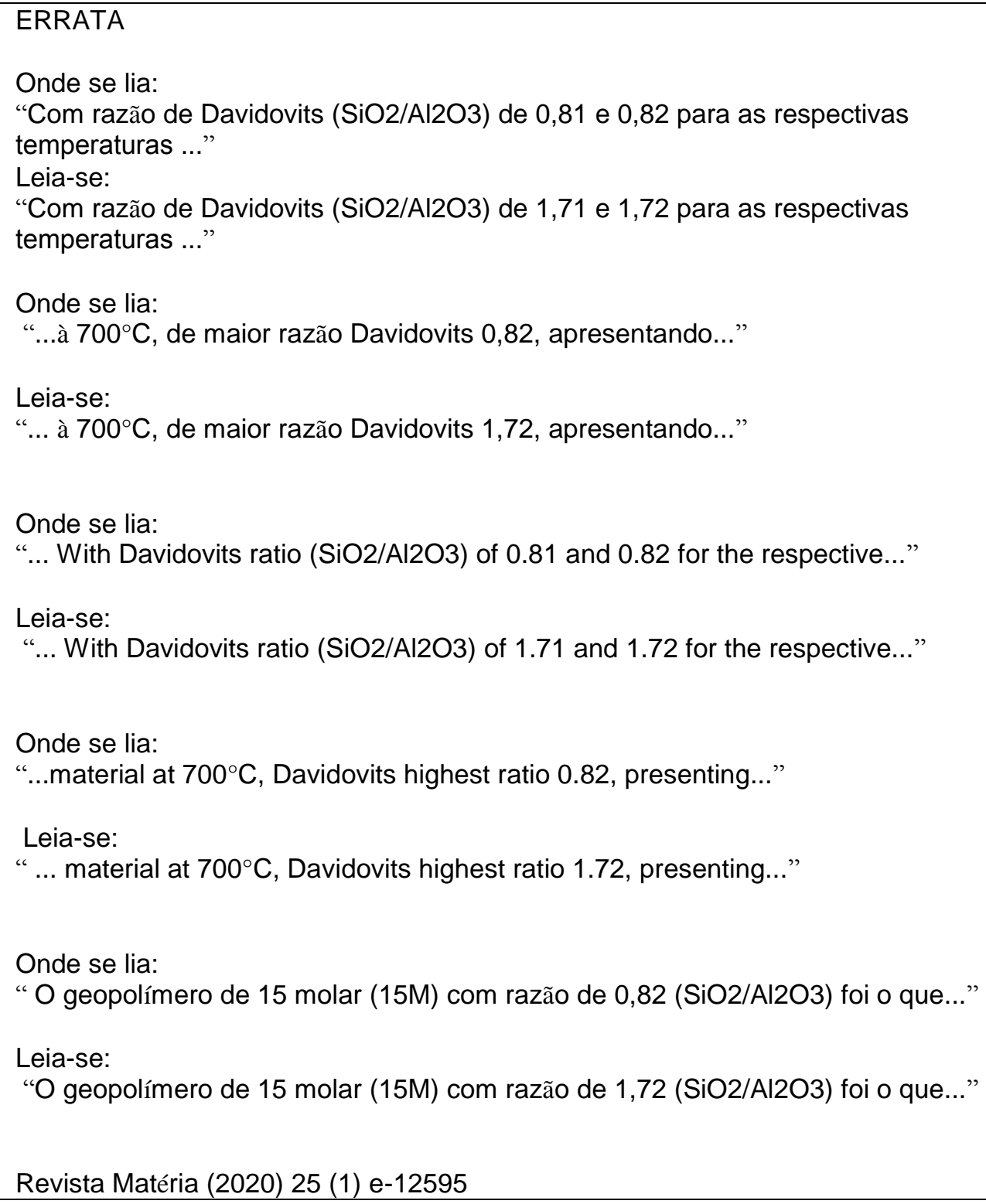

